\title{
SIMULTANEOUS DETERMINATION OF DISTANCE BETWEEN SETS BY MULTIVALUED KANNAN TYPE COUPLING
}

\section{BINAYAK S. CHOUDHURY ${ }^{1}$, PRANATI MAITY ${ }^{2}$, NIKHILESH METIYA ${ }^{3}$ AND MIHAI POSTOLACHE ${ }^{4,5,6, *}$}

${ }^{1}$ Department of Mathematics, Indian Institute of Engineering Science and Technology, Shibpur, Howrah 711103, West Bengal, India

${ }^{2}$ Department of Mathematics, National Institute of Technology, Rourkela, Rourkela 769008, Odisha, India

${ }^{3}$ Department of Mathematics, Sovarani Memorial College, Jagatballavpur, Howrah 711408, West Bengal, India

${ }^{4}$ Department of General Education, China Medical University, Taichung 40402, Taiwan

${ }^{5}$ Gh. Mihoc-C. Iacob Institute of Mathematical Statistics and Applied Mathematics of the Romanian Academy, Bucharest 050711, Romania

${ }^{6}$ Department of Mathematics 83 Computer Science, University Politehnica of Bucharest, 313 Splaiul Independenţei, Bucharest 060042, Romania

*Corresponding author: mi.postolache@mail.cmuh.org.tw; mihai@mathem.pub.ro

Received 2018-04-22; accepted 2018-06-23; published 2018-09-05.

2010 Mathematics Subject Classification. 47H10, 54H25.

Key words and phrases. multivalued coupling; coulpled best proximity point; metric spaces; uniformly convex Banach space. (C)2018 Authors retain the copyrights of their papers, and all open access articles are distributed under the terms of the Creative Commons Attribution License. 
Abstract. In this paper we define a multivalued Kannan type coupling between two subsets of a metric space and use it to obtain the distance between the two subsets through the determination of two pairs of points simultaneously. The problem is a multivalued coupled proximity point problem which falls under the general category of global optimization and is approached from the standpoint of fixed point theory. We use UC-property which is a geometric property that holds automatically for appropriate pairs of subsets of uniformly convex Banach spaces and is adapted to metric spaces by certain postulations. The main results are illustrated with examples. Corresponding results are obtained in Banach spaces. The work is in the domain of setvalued analysis.

\section{INTRODUCTION}

The purpose of the present work is to find algorithmically the distance between two sets through the determination of two pairs of points simultaneously for which we use a multivalued coupling between the two sets. We define the coupling here. We assume that the multivalued coupling satisfies a Kannan-type inequality in which $\delta$-distance is used. It is a new approach to the global optimization problem known as best proximity point problem which seeks to determine the distance between two sets with the help of mappings defined between them. The problem is treated as that of finding an optimal approximate solution of a fixed point equation. It was introduced in the work of Eldered [15] and has been considered by several researchers subsequently. Singlevalued mappings were originally used in these problems. Later multivalued functions were also utilized. Some instances of works on best proximity point problems using single valued mappings are $[1,6-8,11,13,20,25,26]$ while use of multivalued functions in these problems appear in works like $[2,12,18,24]$. Here we have a different approach to the problem mentioned above in which we use a coupled mapping between two sets. Essentially we find an optimal approximate solution to coupled fixed point equations which allows us to determine simultaneously the required distance through two different pairs of points. Moreover we use multivalued coupling. The coupling is assumed to satisfy an inequality which is of Kannan type. Kannan type inequalities are different from Banach's contraction inequality and has very different properties including the possibility that the mappings satisfying these inequalities may be discontinuous. These inequalities first appeared in the work of Kannan [21,22] and has been discussed in a large number of subsequent works $[10,14,16,19,23]$.

\section{Mathematical Preliminaries}

Throughout this paper $(X, d)$ stands for a general metric space. Let $A$ and $B$ be two subsets of $X$. A pair of points $(a, b)$ with $a \in A$ and $b \in B$ is said to be a best proximity pair if $d(a, b)=d(A, B)$ where $d(A, B)=\inf \{d(x, y): x \in A$ and $y \in B\}$. If $T: A \longrightarrow B$ is a non-self mapping for which there exists $z \in A$ such that $d(z, T z)=d(A, B)$, that is, where $(z, T z)$ is a best proximity pair, we say that $z$ is a 
best proximity point of $T$ and the problem of finding such a point is known as the best proximity point problem. It is a global optimization problem in that it seeks to find a point $z \in A$ which minimizes the value of $d(z, T z)$ over $z \in A$ subject to the condition that the minimum is $d(A, B)$. On the other hand it is an extension of the fixed point problem and reduces to that problem in the special case where $A \cap B \neq \phi$.

The following are the concepts from setvalued analysis which we shall use in this paper. Let $(X, d)$ be a metric space. Let $N(X):=$ the collection of all non-empty subsets of $X ; B(X):=$ the collection of all non-empty bounded subsets of $X$ and $C B(X):=$ the collection of all non-empty closed and bounded subsets of $X$. Now for $A, B \in B(X)$, the functions $D$ and $\delta$ are defined as follows.

$$
D(A, B)=\inf \{d(a, b): a \in A, b \in B\} \text { and } \delta(A, B)=\sup \{d(a, b): a \in A, b \in B\}
$$

$D(A, B)$ is sometimes denoted as $d(A, B)$ or $\operatorname{dist}(A, B)$. If $A=\{a\}$, then we write $D(A, B)=D(a, B)$ and $\delta(A, B)=\delta(a, B)$. Also in addition, if $B=\{b\}$, then $D(A, B)=d(a, b)$ and $\delta(A, B)=d(a, b)$. For $A, B, C \in B(X)$, the definition of $\delta(A, B)$ yields the following:

$$
\begin{aligned}
& \delta(A, B)=\delta(B, A) ; \quad \delta(A, B) \leq \delta(A, C)+\delta(C, B) ; \\
& \delta(A, B)=0 \text { iff } A=B=\{a\} ; \quad \delta(A, A)=\operatorname{diam} A[17] .
\end{aligned}
$$

The $\delta$-distance has all the properties of a metric except one. It has been used in works like $[4,5,9]$. We use this concept in our theorem.

Let $A$ and $B$ be two non-empty subsets of a metric space $(X, d)$ and $T: A \rightarrow C B(B)$ a multivalued mapping. A point $x^{*} \in A$ is called a best proximity point of $T$ if $D\left(x^{*}, T x^{*}\right)=d(A, B)$ [2]. This is a natural generalization of its singlevalued counterpart described in the above. A fixed point $x$ of a multivalued mapping $T$ is given by the following inclusion relation $x \in T x$. Now there need not be a fixed point of the multivalued mapping in general. Here the task in the best proximity point problem is to find a global minima of the function $x \mapsto D(x, T x)$ by constraining an approximate solution of the inclusion relation $x \in T x$ to satisfy $D(x, T x)=d(A, B)$.

Here we take another approach to the above problem. For that purpose we define singlevalued and multivalued coupling functions.

Definition 2.1. Let $A$ and $B$ be two non-empty subsets of a metric space $(X, d)$. A mapping $F: X \times X \longrightarrow$ $X$ is said to be a coupling with respect to $A$ and $B$ if $F(x, y) \in B$ for $(x, y) \in A \times B$ and $F(x, y) \in A$ for $(x, y) \in B \times A$.

Definition 2.2. Let $A$ and $B$ be two non-empty subsets of a metric space $(X, d)$. A multivalued mapping $F: X \times X \longrightarrow N(X)$ is said to be a multivalued coupling with respect to $A$ and $B$ if $F(x, y) \subseteq B$ for $(x, y) \in A \times B$ and $F(x, y) \subseteq A$ for $(x, y) \in B \times A$. 
Definition 2.3 (Property UC [26]). Let $A$ and $B$ be two non-empty subsets of a metric space $(X, d)$. Then the pair $(A, B)$ is said to satisfy the property $\mathrm{UC}$ if for sequences $\left\{x_{n}\right\}$ and $\left\{x_{n}^{\prime}\right\}$ in $A$ and $\left\{y_{n}\right\}$ in $B$,

$$
\lim _{n \longrightarrow \infty} d\left(x_{n}, y_{n}\right)=d(A, B) \text { and } \lim _{n \longrightarrow \infty} d\left(x_{n}^{\prime}, y_{n}\right)=d(A, B) \Longrightarrow \lim _{n \longrightarrow \infty} d\left(x_{n}, x_{n}^{\prime}\right)=0
$$

Lemma 2.1 ( [26]). Let $A$ and $B$ be two non-empty subsets of a metric space $(X, d)$. Assume that the pair $(A, B)$ has the property UC. Let $\left\{x_{n}\right\}$ and $\left\{y_{n}\right\}$ be sequences in $A$ and $B$ respectively such that either of the following holds:

$$
\lim _{m \longrightarrow \infty} \sup _{n \geq m} d\left(x_{m}, y_{n}\right)=d(A, B) \quad \text { or } \lim _{n \longrightarrow \infty} \sup _{m \geq n} d\left(x_{m}, y_{n}\right)=d(A, B) .
$$

Then $\left\{x_{n}\right\}$ is a Cauchy sequence.

Our purpose here is to realize the minimum distance between two sets $A$ and $B$ through a coupled best proximity point for singlevalued and multivalued coupling which we define below.

Definition 2.4. Let $A$ and $B$ be two non-empty subsets of a metric space $(X, d)$ and $F: X \times X \longrightarrow X$ be a coupling with respect to $A$ and $B$. An element $(x, y) \in A \times B$ is called a coupled best proximity point of $F$ if $d(x, F(x, y))=d(A, B)$ and $d(y, F(y, x))=d(A, B)$.

Definition 2.5. Let $A$ and $B$ be two non-empty subsets of a metric space $(X, d)$ and $F: X \times X \longrightarrow N(X)$ be a multivalued coupling with respect to $A$ and $B$. An element $(x, y) \in A \times B$ is called a coupled best proximity point of $F$ if $D(x, F(x, y))=d(A, B)$ and $D(y, F(y, x))=d(A, B)$.

It is noted that proximity points for coupled mapping have been defined in [20]. Our definition is for both singlevalued and multivalued couplings which is different from the above mentioned concept. Also the present work is in a different course from that of [20].

Here the problem of finding coupled best proximity points for multivalued couplings is viewed as that of finding simultaneous optimal approximate solutions of the coupled fixed point inclusions $x \in F(x, y)$ and $y \in F(y, x)$ for a multivalued coupling $F$ in the set $A \times B$ such that the solution satisfies $D(x, F(x, y))=$ $D(y, F(y, x))=d(A, B)$. In general, the exact solutions may not exist. This is surely the case where $d(A, B) \neq 0$ which is of interest here. From another viewpoint, it is the problem of simultaneous minimization of $D(x, F(x, y))$ and $D(y, F(y, x))$ for $x \in A, y \in B$ such that the minimum values at the point of optimality are the global minimum $d(A, B)$. It is to be noted that the pairs $(x, F(x, y))$ and $(y, F(y, x))$ are in general different. Thus the distance between the two sets is obtained through two different pairs of points, that is, in the process we obtain two best proximity pairs. Also the coupled best proximity point may not be unique.

We discuss an application of our main result in uniformly convex Banach spaces. 
Definition 2.6 ( [3]). A Banach space $X$ is said to be uniformly convex if for every $\epsilon$ satisfying $0<\epsilon \leq 2$ there corresponds a $\delta(\epsilon)>0$ such that the conditions hold for all $x, y \in X$,

$$
\|x\|=\|y\|=1 \text { and }\|x-y\| \geq \epsilon \Longrightarrow\left\|\frac{x+y}{2}\right\| \leq 1-\delta(\epsilon)
$$

We use the following results in our application.

Lemma 2.2 ( [15]). Let $A$ be a non-empty closed and convex subset and $B$ be a non-empty closed subset of a uniformly convex Banach space. Let $\left\{x_{n}\right\}$ and $\left\{z_{n}\right\}$ be sequences in $A$ and $\left\{y_{n}\right\}$ be a sequence in $B$ such that (i) $\left\|z_{n}-y_{n}\right\| \longrightarrow d(A, B)$ as $n \longrightarrow \infty$ and (ii) for every $\epsilon>0$ there exists $N_{0}$ such that for all $m>n \geq N_{0},\left\|x_{m}-y_{n}\right\| \leq d(A, B)+\epsilon$. Then for every $\epsilon>0$ there exists $N_{1}$ such that for all $m>n \geq N_{1}$, $\left\|x_{m}-z_{n}\right\| \leq \epsilon$.

Lemma 2.3 ( [15]). Let $A$ be a non-empty closed and convex subset and $B$ be a non-empty closed subset of a uniformly convex Banach space. Let $\left\{x_{n}\right\}$ and $\left\{z_{n}\right\}$ be sequences in $A$ and $\left\{y_{n}\right\}$ be a sequence in $B$ such that (i) $\left\|x_{n}-y_{n}\right\| \longrightarrow d(A, B)$ as $n \longrightarrow \infty$ and (ii) $\left\|z_{n}-y_{n}\right\| \longrightarrow d(A, B)$ as $n \longrightarrow \infty$. Then $\left\|x_{n}-z_{n}\right\| \longrightarrow 0$ as $n \longrightarrow \infty$.

Here in our theorem we have established the existence of a coupled proximity points of a multivalued coupling in a metric space $(X, d)$ which satisfies certain coupled inequality. The theorems are illustrated with examples.

\section{Main Results}

Theorem 3.1. Let $A$ and $B$ be two non-empty closed subsets of a complete metric space $(X, d)$ and $F: X \times X \longrightarrow B(X)$ be a coupling with respect to $A$ and $B$. Suppose there exists $k \in\left[0, \frac{1}{2}\right)$ such that for $x, v \in A$ and $y, u \in B$, the following inequality holds

$$
\delta(F(x, y), F(u, v)) \leq k[D(x, F(x, y))+D(u, F(u, v))]+(1-2 k) d(A, B)
$$

Then there exist two sequences $\left\{x_{n}\right\}$ and $\left\{y_{n}\right\}$ in $A$ and $B$ respectively such that $\lim _{n \longrightarrow \infty} d\left(x_{n}, y_{n+1}\right)=$ $d(A, B)$ and $\lim _{n \longrightarrow \infty} d\left(y_{n}, x_{n+1}\right)=d(A, B)$. Further, if $\left\{x_{n}\right\}$ and $\left\{y_{n}\right\}$ are Cauchy sequences, then $F$ has a coupled best proximity point.

Proof. Starting with $x_{0} \in A$ and $y_{0} \in B$, we construct two sequences $\left\{x_{n}\right\}$ and $\left\{y_{n}\right\}$ respectively in $A$ and $B$ as follows

$$
x_{n+1} \in F\left(y_{n}, x_{n}\right) \text { and } y_{n+1} \in F\left(x_{n}, y_{n}\right) \text { for all } n \geq 0 \text {. }
$$


By (3.1) and (3.2), we have

$$
\begin{aligned}
d\left(x_{1}, y_{2}\right) & \leq \delta\left(F\left(y_{0}, x_{0}\right), F\left(x_{1}, y_{1}\right)\right)=\delta\left(F\left(x_{1}, y_{1}\right), F\left(y_{0}, x_{0}\right)\right) \\
& \leq k\left[D\left(x_{1}, F\left(x_{1}, y_{1}\right)\right)+D\left(y_{0}, F\left(y_{0}, x_{0}\right)\right)\right]+(1-2 k) d(A, B) \\
& \leq k\left[d\left(x_{1}, y_{2}\right)+d\left(y_{0}, x_{1}\right)\right]+(1-2 k) d(A, B),
\end{aligned}
$$

which implies that

$$
d\left(x_{1}, y_{2}\right) \leq \frac{k}{1-k} d\left(y_{0}, x_{1}\right)+\frac{1-2 k}{1-k} d(A, B)
$$

Put $t=\frac{k}{1-k}$. Then $0 \leq t<1$ and the above inequality becomes

$$
d\left(x_{1}, y_{2}\right) \leq t d\left(y_{0}, x_{1}\right)+(1-t) d(A, B) .
$$

Again, using (3.1) and (3.2), we have

$$
\begin{aligned}
d\left(y_{1}, x_{2}\right) & \leq \delta\left(F\left(x_{0}, y_{0}\right), F\left(y_{1}, x_{1}\right)\right) \\
& \leq k\left[D\left(x_{0}, F\left(x_{0}, y_{0}\right)\right)+D\left(y_{1}, F\left(y_{1}, x_{1}\right)\right)\right]+(1-2 k) d(A, B) \\
& \leq k\left[d\left(x_{0}, y_{1}\right)+d\left(y_{1}, x_{2}\right)\right]+(1-2 k) d(A, B)
\end{aligned}
$$

which implies that

$$
\begin{aligned}
d\left(y_{1}, x_{2}\right) & \leq \frac{k}{1-k} d\left(x_{0}, y_{1}\right)+\frac{1-2 k}{1-k} d(A, B) \\
& =t d\left(x_{0}, y_{1}\right)+(1-t) d(A, B) .
\end{aligned}
$$

Using (3.1) and (3.2), we have

$$
\begin{aligned}
d\left(x_{2}, y_{3}\right) & \leq \delta\left(F\left(y_{1}, x_{1}\right), F\left(x_{2}, y_{2}\right)\right)=\delta\left(F\left(x_{2}, y_{2}\right), F\left(y_{1}, x_{1}\right)\right) \\
& \leq k\left[D\left(x_{2}, F\left(x_{2}, y_{2}\right)\right)+D\left(y_{1}, F\left(y_{1}, x_{1}\right)\right)\right]+(1-2 k) d(A, B) \\
& \leq k\left[d\left(x_{2}, y_{3}\right)+d\left(y_{1}, x_{2}\right)\right]+(1-2 k) d(A, B),
\end{aligned}
$$

that is,

$$
\begin{aligned}
d\left(x_{2}, y_{3}\right) & \leq \frac{k}{1-k} d\left(y_{1}, x_{2}\right)+\left(1-\frac{k}{1-k}\right) d(A, B) \\
& =t d\left(y_{1}, x_{2}\right)+(1-t) d(A, B) \\
& \leq t\left[t d\left(x_{0}, y_{1}\right)+(1-t) d(A, B)\right]+(1-t) d(A, B) \quad[b y(3.4)] \\
& =t^{2} d\left(x_{0}, y_{1}\right)+\left(1-t^{2}\right) d(A, B) .
\end{aligned}
$$


Using (3.1) and (3.2), we have

$$
\begin{aligned}
d\left(y_{2}, x_{3}\right) & \leq \delta\left(F\left(x_{1}, y_{1}\right), F\left(y_{2}, x_{2}\right)\right) \\
& \leq k\left[D\left(x_{1}, F\left(x_{1}, y_{1}\right)\right)+D\left(y_{2}, F\left(y_{2}, x_{2}\right)\right)\right]+(1-2 k) d(A, B) \\
& \leq k\left[d\left(x_{1}, y_{2}\right)+d\left(y_{2}, x_{3}\right)\right]+(1-2 k) d(A, B),
\end{aligned}
$$

that is,

$$
\begin{aligned}
d\left(y_{2}, x_{3}\right) & \leq \frac{k}{1-k} d\left(x_{1}, y_{2}\right)+\left(1-\frac{k}{1-k}\right) d(A, B) \\
& =t d\left(x_{1}, y_{2}\right)+(1-t) d(A, B) \\
& \leq t\left[t d\left(y_{0}, x_{1}\right)+(1-t) d(A, B)\right]+(1-t) d(A, B) \quad[b y(3.3)] \\
& =t^{2} d\left(y_{0}, x_{1}\right)+\left(1-t^{2}\right) d(A, B)
\end{aligned}
$$

Again, using (3.1) and (3.2), we have

$$
\begin{aligned}
d\left(x_{3}, y_{4}\right) & \leq \delta\left(F\left(y_{2}, x_{2}\right), F\left(x_{3}, y_{3}\right)\right)=\delta\left(F\left(x_{3}, y_{3}\right), F\left(y_{2}, x_{2}\right)\right) \\
& \leq k\left[D\left(x_{3}, F\left(x_{3}, y_{3}\right)\right)+D\left(y_{2}, F\left(y_{2}, x_{2}\right)\right)\right]+(1-2 k) d(A, B) \\
& \leq k\left[d\left(x_{3}, y_{4}\right)+d\left(y_{2}, x_{3}\right)\right]+(1-2 k) d(A, B)
\end{aligned}
$$

that is,

$$
\begin{aligned}
d\left(x_{3}, y_{4}\right) & \leq \frac{k}{1-k} d\left(y_{2}, x_{3}\right)+\left(1-\frac{k}{1-k}\right) d(A, B) \\
& =t d\left(y_{2}, x_{3}\right)+(1-t) d(A, B) \\
& \leq t\left[t^{2} d\left(y_{0}, x_{1}\right)+\left(1-t^{2}\right) d(A, B)\right]+(1-t) d(A, B) \quad[b y(3.6)] \\
& =t^{3} d\left(y_{0}, x_{1}\right)+\left(1-t^{3}\right) d(A, B) .
\end{aligned}
$$

And

$$
\begin{aligned}
d\left(y_{3}, x_{4}\right) & \leq \delta\left(F\left(x_{2}, y_{2}\right), F\left(y_{3}, x_{3}\right)\right) \\
& \leq k\left[D\left(x_{2}, F\left(x_{2}, y_{2}\right)\right)+D\left(y_{3}, F\left(y_{3}, x_{3}\right)\right)\right]+(1-2 k) d(A, B) \\
& \leq k\left[d\left(x_{2}, y_{3}\right)+d\left(y_{3}, x_{4}\right)\right]+(1-2 k) d(A, B),
\end{aligned}
$$


that is,

$$
\begin{aligned}
d\left(y_{3}, x_{4}\right) & \leq \frac{k}{1-k} d\left(x_{2}, y_{3}\right)+\left(1-\frac{k}{1-k}\right) d(A, B) \\
& =t d\left(x_{2}, y_{3}\right)+(1-t) d(A, B) \\
& \leq t\left[t^{2} d\left(x_{0}, y_{1}\right)+\left(1-t^{2}\right) d(A, B)\right]+(1-t) d(A, B) \quad[b y(3.5)] \\
& =t^{3} d\left(x_{0}, y_{1}\right)+\left(1-t^{3}\right) d(A, B) .
\end{aligned}
$$

Let $m$ be any positive integer.

Assume that if $n$ is odd and $n \leq m$, then

$$
d\left(x_{n}, y_{n+1}\right) \leq t^{n} d\left(y_{0}, x_{1}\right)+\left(1-t^{n}\right) d(A, B)
$$

and

$$
d\left(y_{n}, x_{n+1}\right) \leq t^{n} d\left(x_{0}, y_{1}\right)+\left(1-t^{n}\right) d(A, B) .
$$

Assume that if $n$ is is even and $n \leq m$, then

$$
d\left(x_{n}, y_{n+1}\right) \leq t^{n} d\left(x_{0}, y_{1}\right)+\left(1-t^{n}\right) d(A, B)
$$

and

$$
d\left(y_{n}, x_{n+1}\right) \leq t^{n} d\left(y_{0}, x_{1}\right)+\left(1-t^{n}\right) d(A, B) .
$$

Let $m$ be even. Then

$$
\begin{aligned}
d\left(x_{m+1}, y_{m+2}\right) & \leq \delta\left(F\left(y_{m}, x_{m}\right), F\left(x_{m+1}, y_{m+1}\right)\right)=\delta\left(F\left(x_{m+1}, y_{m+1}\right), F\left(y_{m}, x_{m}\right)\right) \\
& \leq k\left[D\left(x_{m+1}, F\left(x_{m+1}, y_{m+1}\right)+D\left(y_{m}, F\left(y_{m}, x_{m}\right)\right)\right)\right]+(1-2 k) d(A, B) \\
& \leq k\left[d\left(x_{m+1}, y_{m+2}\right)+d\left(y_{m}, x_{m+1}\right)\right]+(1-2 k) d(A, B),
\end{aligned}
$$

that is,

$$
\begin{aligned}
d\left(x_{m+1}, y_{m+2}\right) & \leq \frac{k}{1-k} d\left(y_{m}, x_{m+1}\right)+\left(1-\frac{k}{1-k}\right) d(A, B) \\
& \leq t\left[t^{m} d\left(y_{0}, x_{1}\right)+\left(1-t^{m}\right) d(A, B)\right]+(1-t) d(A, B)(b y(3.12)) \\
& \leq t^{m+1} d\left(y_{0}, x_{1}\right)+\left(1-t^{m+1}\right) d(A, B) .
\end{aligned}
$$

And

$$
\begin{aligned}
d\left(y_{m+1}, x_{m+2}\right) & \leq \delta\left(F\left(x_{m}, y_{m}\right), F\left(y_{m+1}, x_{m+1}\right)\right) \\
& \leq k\left[D\left(x_{m}, F\left(x_{m}, y_{m}\right)\right)+D\left(y_{m+1}, F\left(y_{m+1}, x_{m+1}\right)\right)\right]+(1-2 k) d(A, B) \\
& \leq k\left[d\left(x_{m}, y_{m+1}\right)+d\left(y_{m+1}, x_{m+2}\right)\right]+(1-2 k) d(A, B),
\end{aligned}
$$


that is,

$$
\begin{aligned}
d\left(y_{m+1}, x_{m+2}\right) & \leq \frac{k}{1-k} d\left(x_{m}, y_{m+1}\right)+\left(1-\frac{k}{1-k}\right) d(A, B) \\
& \leq t\left[t^{m} d\left(x_{0}, y_{1}\right)+\left(1-t^{m}\right) d(A, B)\right]+(1-t) d(A, B)(b y(3.11)) \\
& \leq t^{m+1} d\left(x_{0}, y_{1}\right)+\left(1-t^{m+1}\right) d(A, B)
\end{aligned}
$$

Let $m$ be odd. Then

$$
\begin{aligned}
d\left(x_{m+1}, y_{m+2}\right) & \leq \delta\left(F\left(y_{m}, x_{m}\right), F\left(x_{m+1}, y_{m+1}\right)\right)=\delta\left(F\left(x_{m+1}, y_{m+1}\right), F\left(y_{m}, x_{m}\right)\right) \\
\leq & k\left[D\left(x_{m+1}, F\left(x_{m+1}, y_{m+1}\right)\right)+D\left(y_{m}, F\left(y_{m}, x_{m}\right)\right)\right]+(1-2 k) d(A, B) \\
\leq & k\left[d\left(x_{m+1}, y_{m+2}\right)+d\left(y_{m}, x_{m+1}\right)\right]+(1-2 k) d(A, B),
\end{aligned}
$$

that is,

$$
\begin{aligned}
d\left(x_{m+1}, y_{m+2}\right) & \leq \frac{k}{1-k} d\left(y_{m}, x_{m+1}\right)+\left(1-\frac{k}{1-k}\right) d(A, B) \\
& \leq t\left[t^{m} d\left(x_{0}, y_{1}\right)+\left(1-t^{m}\right) d(A, B)\right]+(1-t) d(A, B)(b y(3.10)) \\
& \leq t^{m+1} d\left(x_{0}, y_{1}\right)+\left(1-t^{m+1}\right) d(A, B) .
\end{aligned}
$$

And

$$
\begin{aligned}
d\left(y_{m+1}, x_{m+2}\right) & \leq \delta\left(F\left(x_{m}, y_{m}\right), F\left(y_{m+1}, x_{m+1}\right)\right) \\
& \leq k\left[D\left(x_{m}, F\left(x_{m}, y_{m}\right)\right)+D\left(y_{m+1}, F\left(y_{m+1}, x_{m+1}\right)\right)\right]+(1-2 k) d(A, B) \\
& \leq k\left[d\left(x_{m}, y_{m+1}\right)+d\left(y_{m+1}, x_{m+2}\right)\right]+(1-2 k) d(A, B),
\end{aligned}
$$

that is,

$$
\begin{aligned}
d\left(y_{m+1}, x_{m+2}\right) & \leq \frac{k}{1-k} d\left(x_{m}, y_{m+1}\right)+\left(1-\frac{k}{1-k}\right) d(A, B) \\
& \leq t\left[t^{m} d\left(y_{0}, x_{1}\right)+\left(1-t^{m}\right) d(A, B)\right]+(1-t) d(A, B)(b y(3.9)) \\
& \leq t^{m+1} d\left(y_{0}, x_{1}\right)+\left(1-t^{m+1}\right) d(A, B) .
\end{aligned}
$$

Thus (3.9)-(3.12) are valid for $m+1$. By induction method, (3.9) and (3.10) are valid for all odd integers $n$ and (3.11) and (3.12) are valid for all even integers $n$. Therefore, we conclude that for all integers $n \geq 0$, we have

$$
d\left(x_{2 n+1}, y_{2 n+2}\right) \leq t^{2 n+1} d\left(y_{0}, x_{1}\right)+\left(1-t^{2 n+1}\right) d(A, B)
$$

and

$$
d\left(x_{2 n}, y_{2 n+1}\right) \leq t^{2 n} d\left(x_{0}, y_{1}\right)+\left(1-t^{2 n}\right) d(A, B) .
$$


Also, for all integer $n \geq 0$, we conclude that

$$
d\left(y_{2 n+1}, x_{2 n+2}\right) \leq t^{2 n+1} d\left(x_{0}, y_{1}\right)+\left(1-t^{2 n+1}\right) d(A, B)
$$

and

$$
d\left(y_{2 n}, x_{2 n+1}\right) \leq t^{2 n} d\left(y_{0}, x_{1}\right)+\left(1-t^{2 n}\right) d(A, B) .
$$

Since $\left\{x_{n}\right\}$ and $\left\{y_{n}\right\}$ sequences respectively in $A$ and $B$ and $0 \leq t<1$, taking $n \longrightarrow \infty$ in (3.13), (3.14), (3.15) and (3.16), we have

$$
\lim _{n \longrightarrow \infty} d\left(x_{2 n}, y_{2 n+1}\right)=\lim _{n \longrightarrow \infty} d\left(x_{2 n+1}, y_{2 n+2}\right)=d(A, B)
$$

and

$$
\lim _{n \longrightarrow \infty} d\left(y_{2 n}, x_{2 n+1}\right)=\lim _{n \longrightarrow \infty} d\left(y_{2 n+1}, x_{2 n+2}\right)=d(A, B) .
$$

Now, (3.17) and (3.18) respectively implies that

$$
\lim _{n \longrightarrow \infty} d\left(x_{n}, y_{n+1}\right)=d(A, B) \text { and } \lim _{n \longrightarrow \infty} d\left(y_{n}, x_{n+1}\right)=d(A, B) .
$$

Further, suppose that $\left\{x_{n}\right\}$ and $\left\{y_{n}\right\}$ are Cauchy sequences. Here $X$ is complete and $A$ and $B$ are closed subsets of $X$. Since $\left\{x_{n}\right\}$ and $\left\{y_{n}\right\}$ are sequences respectively in $A$ and $B$, there exist $x \in A$ and $y \in B$ such that

$$
x_{n} \longrightarrow x \text { and } y_{n} \longrightarrow y \text { as } n \longrightarrow \infty \text {. }
$$

By (3.19) and (3.20), we have

$$
\lim _{n \longrightarrow \infty} d\left(x_{n}, y_{n+1}\right)=d(x, y)=d(A, B)
$$

and

$$
\lim _{n \longrightarrow \infty} d\left(y_{n}, x_{n+1}\right)=d(y, x)=d(x, y)=d(A, B) .
$$

Since $F$ is coupling with respect to $A$ and $B$, and $x \in A, y \in B$, we have $F(x, y) \subseteq B$. Then we have

$$
d(A, B) \leq D(x, F(x, y))
$$

Using (3.1) and (3.2), we obtain

$$
\begin{aligned}
D(x, F(x, y)) & \leq \delta(x, F(x, y)) \leq d\left(x, x_{n+1}\right)+\delta\left(x_{n+1}, F(x, y)\right) \\
& \leq d\left(x, x_{n+1}\right)+\delta\left(F\left(y_{n}, x_{n}\right), F(x, y)\right) \\
& =d\left(x, x_{n+1}\right)+\delta\left(F(x, y), F\left(y_{n}, x_{n}\right)\right) \\
& \leq d\left(x, x_{n+1}\right)+k\left[D(x, F(x, y))+D\left(y_{n}, F\left(y_{n}, x_{n}\right)\right)\right]+(1-2 k) d(A, B) \\
& \leq d\left(x, x_{n+1}\right)+k\left[D(x, F(x, y))+d\left(y_{n}, x_{n+1}\right)\right]+(1-2 k) d(A, B),
\end{aligned}
$$


which implies that

$$
D(x, F(x, y)) \leq \frac{1}{1-k} d\left(x, x_{n+1}\right)+\frac{k}{1-k} d\left(y_{n}, x_{n+1}\right)+\left(1-\frac{k}{1-k}\right) d(A, B) .
$$

Taking $n \longrightarrow \infty$ in the above inequality, using (3.20) and (3.22), we have

$$
D(x, F(x, y)) \leq \frac{k}{1-k} d(A, B)+\left(1-\frac{k}{1-k}\right) d(A, B)=d(A, B) .
$$

Combining (3.23) and (3.24), we have

$$
d(A, B) \leq D(x, F(x, y)) \leq d(A, B)
$$

which implies $D(x, F(x, y))=d(A, B)$.

Similarly, we can prove that $D(y, F(y, x))=d(A, B)$. Hence we have $D(x, F(x, y))=d(A, B)$ and $D(y, F(y, x))=d(A, B)$, that is, $(x, y)$ is a coupled best proximity point of $F$.

Example 3.1. Let $X=C[0, \pi]$ with Chebyshev metric or sup metric. Let $A=\left\{f_{r} \in X: 0 \leq r \leq 1\right\}$ and $B=\left\{g_{r} \in X: 0 \leq r \leq 1\right\}$, where $f_{r}(x)=1-r \sin x$ and $g_{r}(x)=r \sin x-1$, for $x \in[0, \pi]$. Let

$$
F: X \times X \longrightarrow B(X), \quad F(u, v)=\left\{\begin{array}{l}
\left\{g_{1}\right\}, \quad \text { if } u \in A \text { and } v \in B \\
\left\{f_{1}\right\}, \quad \text { if } u \in B \text { and } v \in A \\
\{u+v\}, \quad \text { otherwise }
\end{array}\right.
$$

Let $k \in\left[0, \frac{1}{2}\right)$ be arbitrary. It is verified that all the conditions of the theorem 3.1 are satisfied and $\left(f_{1}, g_{1}\right)$ is a coupled best proximity point of $F$.

In the next theorem we show that in case when the pair $(A, B)$ satisfies the property UC, the sequences $\left\{x_{n}\right\}$ and $\left\{y_{n}\right\}$ constructed in Theorem 3.1 actually converge to the coupled best proximity point of the Kannan type coupling.

Theorem 3.2. Let $(X, d)$ be a complete metric space and $(A, B)$ be a pair of non-empty closed subsets of $X$ satisfying the property UC. Let $F: X \times X \longrightarrow B(X)$ be a coupling with respect to $A$ and $B$ such that (3.1) is satisfied. Then there exist two sequences $\left\{x_{n}\right\}$ and $\left\{y_{n}\right\}$ in $A$ and $B$ respectively such that $\lim _{n \longrightarrow \infty} d\left(x_{n}, y_{n+1}\right)=d(A, B)$ and $\lim _{n \longrightarrow \infty} d\left(y_{n}, x_{n+1}\right)=d(A, B)$. Also, $F$ has a coupled best proximity point.

Proof. We take the same sequences $\left\{x_{n}\right\}$ and $\left\{y_{n}\right\}$ as in Theorem 3.1. Then, $\lim _{n \longrightarrow \infty} d\left(x_{n}, y_{n+1}\right)=d(A, B)$ and $\lim _{n \longrightarrow \infty} d\left(y_{n}, x_{n}\right.$ $d(A, B)$ follows from Theorem 3.1. So we have

$$
\lim _{n \longrightarrow \infty} d\left(x_{2 n}, y_{2 n+1}\right)=d(A, B) \text { and } \lim _{n \longrightarrow \infty} d\left(y_{2 n+1}, x_{2 n+2}\right)=d\left(x_{2 n+2}, y_{2 n+1}\right)=d(A, B) .
$$

Using the property UC, we have

$$
\lim _{n \rightarrow \infty} d\left(x_{2 n}, x_{2 n+2}\right)=0,
$$


Again,

$$
\lim _{n \longrightarrow \infty} d\left(y_{2 n}, x_{2 n+1}\right)=d(A, B) \text { and } \lim _{n \longrightarrow \infty} d\left(x_{2 n+1}, y_{2 n+2}\right)=d\left(y_{2 n+2}, x_{2 n+1}\right)=d(A, B) .
$$

Using the property UC, we have

$$
\lim _{n \longrightarrow \infty} d\left(y_{2 n}, y_{2 n+2}\right)=0
$$

From (3.25) and (3.26), we have that

$$
\lim _{n \longrightarrow \infty} d\left(x_{2 n}, x_{2 n+2}\right)=\lim _{n \longrightarrow \infty} d\left(y_{2 n}, y_{2 n+2}\right)=0 .
$$

Next we prove that $\left\{x_{2 n}\right\}$ and $\left\{y_{2 n}\right\}$ are Cauchy sequences.

We first establish that given $\epsilon>0$ we can find a positive integer $N$ such that for all $m, n>N$,

$$
\max \left\{d\left(x_{2 m}, y_{2 n}\right), d\left(y_{2 m}, x_{2 n}\right)\right\}<d(A, B)+\epsilon
$$

If (3.28) is not valid, then there exists an $\epsilon>0$ for which we can find two sequences of positive integers $\{2 m(p)\}$ and $\{2 n(p)\}$ such that for all positive integers $p$,

$$
\begin{gathered}
2 n(p)>2 m(p)>p \\
\max \left\{d\left(x_{2 m(p)}, y_{2 n(p)}\right), d\left(y_{2 m(p)}, x_{2 n(p)}\right)\right\} \geq d(A, B)+\epsilon
\end{gathered}
$$

and

$$
\max \left\{d\left(x_{2 m(p)}, y_{2 n(p)-2}\right), d\left(y_{2 m(p)}, x_{2 n(p)-2}\right)\right\}<d(A, B)+\epsilon
$$

Then

$$
\begin{aligned}
d(A, B)+\epsilon \quad \leq \max \left\{d\left(x_{2 m(p)}, y_{2 n(p)}\right), d\left(y_{2 m(p)}, x_{2 n(p)}\right)\right\} \\
\leq \max \left\{d\left(x_{2 m(p)}, y_{2 n(p)-2}\right)+d\left(y_{2 n(p)-2}, y_{2 n(p)}\right),\right. \\
\left.d\left(y_{2 m(p)}, x_{2 n(p)-2}\right)+d\left(x_{2 n(p)-2}, x_{2 n(p)}\right)\right\} \\
\leq \max \left\{d\left(x_{2 m(p)}, y_{2 n(p)-2}\right), d\left(y_{2 m(p)}, x_{2 n(p)-2}\right)\right\} \\
\quad+\max \left\{d\left(y_{2 n(p)-2}, y_{2 n(p)}\right), d\left(x_{2 n(p)-2}, x_{2 n(p)}\right)\right\} \\
<d(A, B)+\epsilon+\max \left\{d\left(y_{2 n(p)-2}, y_{2 n(p)}\right), d\left(x_{2 n(p)-2}, x_{2 n(p)}\right)\right\} .
\end{aligned}
$$

Taking $p \longrightarrow \infty$ and using (3.27), we obtain

$$
\lim _{p \longrightarrow \infty} \max \left\{d\left(x_{2 m(p)}, y_{2 n(p)}\right), d\left(y_{2 m(p)}, x_{2 n(p)}\right)\right\}=d(A, B)+\epsilon
$$


Again,

$$
\begin{aligned}
& \max \left\{d\left(x_{2 m(p)}, y_{2 n(p)}\right), d\left(y_{2 m(p)}, x_{2 n(p)}\right)\right\} \\
& \leq \max \left\{\delta\left(F\left(y_{2 m(p)-1}, x_{2 m(p)-1}\right), F\left(x_{2 n(p)-1}, y_{2 n(p)-1}\right)\right),\right. \\
& \left.\quad \delta\left(F\left(x_{2 m(p)-1}, y_{2 m(p)-1}\right), F\left(y_{2 n(p)-1}, x_{2 n(p)-1}\right)\right)\right\} \\
& =\max \left\{\delta\left(F\left(x_{2 n(p)-1}, y_{2 n(p)-1}\right), F\left(y_{2 m(p)-1}, x_{2 m(p)-1}\right)\right),\right. \\
& \left.\quad \delta\left(F\left(x_{2 m(p)-1}, y_{2 m(p)-1}\right), F\left(y_{2 n(p)-1}, x_{2 n(p)-1}\right)\right)\right\} \\
& \leq \max \left\{k\left[D\left(x_{2 n(p)-1}, F\left(x_{2 n(p)-1}, y_{2 n(p)-1}\right)\right)+D\left(y_{2 m(p)-1}, F\left(y_{2 m(p)-1}, x_{2 m(p)-1}\right)\right)\right]\right. \\
& \quad+(1-2 k) d(A, B), \\
& \quad k\left[D\left(x_{2 m(p)-1}, F\left(x_{2 m(p)-1}, y_{2 m(p)-1}\right)\right)+D\left(y_{2 n(p)-1}, F\left(y_{2 n(p)-1}, x_{2 n(p)-1}\right)\right)\right] \\
& \quad+(1-2 k) d(A, B)\} \\
& \leq \max \left\{k\left[d\left(x_{2 n(p)-1}, y_{2 n(p)}\right)+d\left(y_{2 m(p)-1}, x_{2 m(p)}\right)\right]+(1-2 k) d(A, B),\right. \\
& \left.k\left[d\left(x_{2 m(p)-1}, y_{2 m(p)}\right)+d\left(y_{2 n(p)-1}, x_{2 n(p)}\right)\right]+(1-2 k) d(A, B)\right\} .
\end{aligned}
$$

Taking $p \rightarrow \infty$, using (3.19) and (3.29), we get $d(A, B)+\epsilon \leq d(A, B)$, which is a contradiction. Therefore, (3.28) holds.

Since $\left\{x_{n}\right\}$ and $\left\{y_{n}\right\}$ are sequences in $A$ and $B$ respectively, we have

$$
d(A, B) \leq d\left(x_{2 m}, y_{2 n}\right) \text { and } d(A, B) \leq d\left(y_{2 m}, x_{2 n}\right) \text { for all } m, n,
$$

and hence

$$
d(A, B) \leq \max \left\{d\left(x_{2 m}, y_{2 n}\right), d\left(y_{2 m}, x_{2 n}\right)\right\} \text { for all } m, n
$$

Then from (3.28), we can write that given any $\epsilon>0$ there exists an integer $N$ such that for all $m, n>N$

$$
d(A, B)-\epsilon<d(A, B) \leq \max \left\{d\left(x_{2 m}, y_{2 n}\right), d\left(y_{2 m}, x_{2 n}\right)\right\}<d(A, B)+\epsilon,
$$

which implies that for all $m, n>N$

$$
d(A, B)-\epsilon<d(A, B) \leq d\left(x_{2 m}, y_{2 n}\right)<d(A, B)+\epsilon,
$$

and

$$
d(A, B)-\epsilon<d(A, B) \leq d\left(y_{2 m}, x_{2 n}\right)<d(A, B)+\epsilon .
$$

Therefore,

$$
\lim _{m, n \longrightarrow \infty} d\left(x_{2 m}, y_{2 n}\right)=d(A, B) \quad \text { and } \quad \lim _{m, n \longrightarrow \infty} d\left(y_{2 m}, x_{2 n}\right)=d(A, B)
$$


From the above double limits we conclude that

$$
\lim _{n \longrightarrow \infty} \sup _{m \geq n} d\left(x_{2 m}, y_{2 n}\right)=d(A, B) \quad \text { or } \quad \lim _{m \longrightarrow \infty_{n \geq m}} \sup _{n} d\left(x_{2 m}, y_{2 n}\right)=d(A, B)
$$

and

$$
\lim _{n \longrightarrow \infty} \sup _{m \geq n} d\left(y_{2 m}, x_{2 n}\right)=d(A, B) \quad \text { or } \quad \lim _{m \longrightarrow \infty} \sup _{n \geq m} d\left(y_{2 m}, x_{2 n}\right)=d(A, B) .
$$

Thus, by lemma 2.1 it follows that $\left\{x_{2 n}\right\}$ and $\left\{y_{2 n}\right\}$ are a Cauchy sequences in $A$ and $B$ respectively. As $A$ and $B$ are closed subsets of the complete metric space $X$, there exist $u \in A$ and $v \in B$ such that

$$
x_{2 n} \longrightarrow u \text { and } y_{2 n} \longrightarrow v \text { as } n \longrightarrow \infty
$$

Since $F$ is coupling with respect to $A$ and $B$, and $u \in A, v \in B$, we have $F(u, v) \subseteq B$. Then we have

$$
d(A, B) \leq D(u, F(u, v))
$$

Now

$$
\begin{aligned}
D(u, F(u, v)) & \leq \delta(u, F(u, v)) \leq d\left(u, x_{2 n}\right)+\delta\left(x_{2 n}, F(u, v)\right) \\
& \leq d\left(u, x_{2 n}\right)+\delta\left(F\left(y_{2 n-1}, x_{2 n-1}\right), F(u, v)\right) \\
& =d\left(u, x_{2 n}\right)+\delta\left(F(u, v), F\left(y_{2 n-1}, x_{2 n-1}\right)\right) \\
& \leq d\left(u, x_{2 n}\right)+k\left[D(u, F(u, v))+D\left(y_{2 n-1}, F\left(y_{2 n-1}, x_{2 n-1}\right)\right)\right]+(1-2 k) d(A, B) \\
& \leq d\left(u, x_{2 n}\right)+k\left[D(u, F(u, v))+d\left(y_{2 n-1}, x_{2 n}\right)\right]+(1-2 k) d(A, B),
\end{aligned}
$$

which implies that

$$
D(u, F(u, v)) \leq \frac{1}{1-k} d\left(u, x_{2 n}\right)+\frac{k}{1-k} d\left(y_{2 n-1}, x_{2 n}\right)+\left(1-\frac{k}{1-k}\right) d(A, B) .
$$

Taking $n \longrightarrow \infty$ in the above inequality, using (3.30) and the first part of the theorem, we have

$$
D(u, F(u, v)) \leq \frac{k}{1-k} d(A, B)+\left(1-\frac{k}{1-k}\right) d(A, B)=d(A, B) .
$$

Combining (3.31) and (3.32), we have

$$
d(A, B) \leq D(u, F(u, v)) \leq d(A, B)
$$

which implies $D(u, F(u, v))=d(A, B)$. Similarly, we can prove that $D(v, F(v, u))=d(A, B)$. Hence we have $D(u, F(u, v))=d(A, B)$ and $D(v, F(v, u))=d(A, B)$, that is, $(u, v)$ is a coupled best proximity point of $F$. 
Example 3.2. Let $X=R^{2}$ with the metric defined as $d((x, y),(u, v))=|x-u|+|y-v|$ for $(x, y),(u, v) \in$ $X$. Let $A=\{0\} \times[0,1]$ and $B=\{1\} \times[0,1]$. Let $F: X \times X \longrightarrow B(X)$ be defined as

$$
F(x, y)=\left\{\begin{array}{l}
\{(1,1)\}, \quad \text { if } x=(0, \alpha) \in A \text { and } y=(1, \beta) \in B \\
\{(0,1)\}, \quad \text { if } x=(1, \beta) \in B \text { and } y=(0, \alpha) \in A \\
\left\{(u, v):|u| \leq x^{2}+y^{2} \text { and }|v| \leq x^{2}+y^{2}\right\}, \quad \text { otherwise }
\end{array}\right.
$$

Let $k \in\left[0, \frac{1}{2}\right)$ be arbitrary. Then all the conditions of Theorem 3.2 are satisfied and $((0,1),(1,1))$ is a coupled best proximity point of $F$.

\section{Application to uniformly convex Banach spaces}

We now discuss the implications of our result in previous section in uniformly convex Banach spaces for the case of single valued couplings. In this section $D(A, B)=\inf \{\|a-b\|: a \in A, b \in B\}$ and the notation $D(A, B)$ is also denoted as $d(A, B)$ or $\operatorname{dist}(A, B)$.

Theorem 4.1. Let $A$ and $B$ be two non-empty closed subsets of a Banach space $X$. Let $F: X \times X \longrightarrow X$ be a coupling with respect to $A$ and $B$ satisfying the following inequality:

$$
\|F(x, y)-F(u, v)\| \leq k[\|x-F(x, y)\|+\|u-F(u, v)\|]+(1-2 k) d(A, B)
$$

where $x, v \in A, y, u \in B$ and $k \in\left[0, \frac{1}{2}\right)$. Then for arbitrary $\left(x_{0}, y_{0}\right) \in A \times B$, the sequences $\left\{x_{n}\right\}$ and $\left\{y_{n}\right\}$ constructed as

$$
x_{n+1}=F\left(y_{n}, x_{n}\right) \text { and } y_{n+1}=F\left(x_{n}, y_{n}\right) \text { for all } n \geq 0
$$

satisfy

$$
\lim _{n \longrightarrow \infty}\left\|x_{n}-y_{n+1}\right\|=d(A, B) \text { and } \lim _{n \longrightarrow \infty}\left\|y_{n}-x_{n+1}\right\|=d(A, B) .
$$

Further, if $\left\{x_{n}\right\}$ and $\left\{y_{n}\right\}$ are Cauchy sequences, then $F$ has a coupled best proximity point.

Proof. $\{x\} \in B(X)$ for every $x \in X$. Define a mapping $T: X \times X \longrightarrow B(X)$ as $T(x, y)=\{F(x, y)\}$, for $x, y \in$ $X$. Then all the conditions of the theorem reduce to the conditions of Theorem 3.1 and hence by application of Theorem 3.1, $F$ has a coupled best proximity point.

Lemma 4.1. Let $F: X \times X \longrightarrow X$, where $X$ is a normed linear space, be a coupling with respect to two subsets $A$ and $B$ of $X$. Suppose there exists $k \in\left[0, \frac{1}{2}\right)$ such that (4.1) is satisfied for all $x, v \in A$ and $y, u \in B$. Then for arbitrary $\left(x_{0}, y_{0}\right) \in A \times B$, the sequences $\left\{x_{n}\right\}$ and $\left\{y_{n}\right\}$ constructed following (4.2) satisfy $\lim _{n \longrightarrow \infty}\left\|x_{n}-y_{n}\right\|=d(A, B)$.

Proof. By Theorem 4.1, it can be proved that the sequences $\left\{x_{n}\right\}$ and $\left\{y_{n}\right\}$ satisfy (4.3), that is,

$$
\lim _{n \longrightarrow \infty}\left\|x_{n}-y_{n+1}\right\|=d(A, B) \text { and } \lim _{n \longrightarrow \infty}\left\|y_{n}-x_{n+1}\right\|=d(A, B) .
$$


By (4.1) and (4.2), we have for $n \geq 1$,

$$
\begin{aligned}
\left\|x_{n}-y_{n}\right\| & =\left\|F\left(y_{n-1}, x_{n-1}\right)-F\left(x_{n-1}, y_{n-1}\right)\right\|=\left\|F\left(x_{n-1}, y_{n-1}\right)-F\left(y_{n-1}, x_{n-1}\right)\right\| \\
& \leq k\left[\left\|x_{n-1}-F\left(x_{n-1}, y_{n-1}\right)\right\|+\left\|y_{n-1}-F\left(y_{n-1}, x_{n-1}\right)\right\|\right]+(1-2 k) d(A, B) \\
& =k\left[\left\|x_{n-1}-y_{n}\right\|+\left\|y_{n-1}-x_{n}\right\|\right]+(1-2 k) d(A, B) .
\end{aligned}
$$

Since $\left\{x_{n}\right\}$ and $\left\{y_{n}\right\}$ are sequences in $A$ and $B$ respectively, we have

$$
d(A, B) \leq\left\|x_{n}-y_{n}\right\| \text { for all } n \geq 1 .
$$

It follows from (4.4) and (4.5) that

$$
d(A, B) \leq\left\|x_{n}-y_{n}\right\| \leq k\left[\left\|y_{n-1}-x_{n}\right\|+\left\|x_{n-1}-y_{n}\right\|\right]+(1-2 k) d(A, B) .
$$

Taking $n \longrightarrow \infty$ in the above inequality using (4.3), we have $\lim _{n \longrightarrow \infty}\left\|x_{n}-y_{n}\right\|=d(A, B)$.

Remark 4.1. The above lemma is also generally valid in metric spaces. It is not necessary to assume $X$ to be a normed linear space. We use this result only in the theorem for uniformly convex Banach spaces. For this reason we assume $X$ to be a normed linear space in particular.

Theorem 4.2. Let $A$ and $B$ be two non-empty closed and convex subsets of a uniformly convex Banach space $X$. Let $F: X \times X \longrightarrow X$ be a coupling with respect to $A$ and $B$. Suppose there exists $k \in\left[0, \frac{1}{2}\right)$ such that (4.1) is satisfied for all $x, v \in A$ and $y, u \in B$. Then $F$ has a coupled best proximity point.

Proof. Let $x_{0} \in A$ and $y_{0} \in B$. We construct two sequences $\left\{x_{n}\right\}$ and $\left\{y_{n}\right\}$ respectively in $A$ and $B$ which satisfy (4.2), that is,

$$
x_{n+1}=F\left(y_{n}, x_{n}\right) \text { and } y_{n+1}=F\left(x_{n}, y_{n}\right) \text { for all } n \geq 0 .
$$

By Theorem 4.1, we have (4.3). Now by (4.3), we have

$$
\lim _{n \longrightarrow \infty}\left\|x_{n}-y_{n+1}\right\|=d(A, B) \text { and } \lim _{n \longrightarrow \infty}\left\|y_{n+1}-x_{n+2}\right\|=\left\|x_{n+2}-y_{n+1}\right\|=d(A, B) .
$$

By application of Lemma 2.3, we have

$$
\lim _{n \longrightarrow \infty}\left\|x_{n}-x_{n+2}\right\|=0
$$

Again, by (4.3), we have

$$
\lim _{n \longrightarrow \infty}\left\|y_{n}-x_{n+1}\right\|=d(A, B) \text { and } \lim _{n \longrightarrow \infty}\left\|x_{n+1}-y_{n+2}\right\|=\left\|y_{n+2}-x_{n+1}\right\|=d(A, B) .
$$

By application of Lemma 2.3, we have

$$
\lim _{n \longrightarrow \infty}\left\|y_{n}-y_{n+2}\right\|=0 .
$$


Next we prove that $\left\{x_{2 n}\right\},\left\{y_{2 n}\right\},\left\{x_{2 n+1}\right\}$ and $\left\{y_{2 n+1}\right\}$ are Cauchy sequences. In the following we consider the case of the sequences $\left\{x_{2 n}\right\}$ and $\left\{y_{2 n}\right\}$.

From (4.6) and (4.7), we have that

$$
\lim _{n \longrightarrow \infty}\left\|x_{2 n}-x_{2 n+2}\right\|=\lim _{n \longrightarrow \infty}\left\|y_{2 n}-y_{2 n+2}\right\|=0 .
$$

We first establish that given $\epsilon>0$ we can find a positive integer $N$ such that for all $m, n>N$,

$$
\max \left\{\left\|x_{2 m}-y_{2 n}\right\|,\left\|y_{2 m}-x_{2 n}\right\|\right\}<d(A, B)+\epsilon
$$

By Lemma 4.1, we have

$$
\lim _{n \longrightarrow \infty}|| x_{2 n}-y_{2 n} \|=d(A, B)
$$

If (4.9) is not valid, then there exists an $\epsilon>0$ for which we can find two sequences of positive integers $\{2 m(p)\}$ and $\{2 n(p)\}$ such that for all positive integers $p$,

$$
\begin{gathered}
2 n(p)>2 m(p)>p \\
\max \left\{\left\|x_{2 m(p)}-y_{2 n(p)}\right\|,\left\|y_{2 m(p)}-x_{2 n(p)}\right\|\right\} \geq d(A, B)+\epsilon
\end{gathered}
$$

and

$$
\max \left\{\left\|x_{2 m(p)}-y_{2 n(p)-2}\right\|,\left\|y_{2 m(p)}-x_{2 n(p)-2}\right\|\right\}<d(A, B)+\epsilon
$$

Then

$$
\begin{aligned}
d(A, B)+\epsilon \quad & \leq \max \left\{\left\|x_{2 m(p)}-y_{2 n(p)}\right\|,\left\|y_{2 m(p)}-x_{2 n(p)}\right\|\right\} \\
& \leq \max \left\{\left\|x_{2 m(p)}-y_{2 n(p)-2}\right\|+\left\|y_{2 n(p)-2}-y_{2 n(p)}\right\|,\right. \\
& \left.\left\|y_{2 m(p)}-x_{2 n(p)-2}\right\|+\left\|x_{2 n(p)-2}-x_{2 n(p)}\right\|\right\} \\
& \leq \max \left\{\left\|x_{2 m(p)}-y_{2 n(p)-2}\right\|,\left\|y_{2 m(p)}-x_{2 n(p)-2}\right\|\right\} \\
& +\max \left\{\left\|y_{2 n(p)-2}-y_{2 n(p)}\right\|,\left\|x_{2 n(p)-2}-x_{2 n(p)}\right\|\right\} \\
& <d(A, B)+\epsilon+\max \left\{\left\|y_{2 n(p)-2}-y_{2 n(p)}\right\|,\left\|x_{2 n(p)-2}-x_{2 n(p)}\right\|\right\} .
\end{aligned}
$$

Taking $p \longrightarrow \infty$ and using (4.8), we obtain

$$
\lim _{p \longrightarrow \infty} \max \left\{\left\|x_{2 m(p)}-y_{2 n(p)}\right\|,\left\|y_{2 m(p)}-x_{2 n(p)}\right\|\right\}=d(A, B)+\epsilon .
$$


Again, for all $p$,

$$
\begin{aligned}
& \max \left\{\left\|x_{2 m(p)+2}-y_{2 n(p)+2}\right\|,\left\|y_{2 m(p)+2}-x_{2 n(p)+2}\right\|\right\} \\
& \leq \max \left\{\left\|x_{2 m(p)+2}-x_{2 m(p)}\right\|+\left\|x_{2 m(p)}-y_{2 n(p)}\right\|+\left\|y_{2 n(p)}-y_{2 n(p)+2}\right\|\right. \\
& \left.\quad\left\|y_{2 m(p)+2}-y_{2 m(p)}\right\|+\left\|y_{2 m(p)}-x_{2 n(p)}\right\|+\left\|x_{2 n(p)}-x_{2 n(p)+2}\right\|\right\} \\
& \leq \max \left\{\left\|x_{2 m(p)}-y_{2 n(p)}\right\|,\left\|x_{2 n(p)}-y_{2 m(p)}\right\|\right\}+ \\
& \max \left\{\left\|x_{2 m(p)+2}-x_{2 m(p)}\right\|+\left\|y_{2 n(p)}-y_{2 n(p)+2}\right\|\right. \\
& \left.\left\|y_{2 m(p)+2}-y_{2 m(p)}\right\|+\left\|x_{2 n(p)}-x_{2 n(p)+2}\right\|\right\}
\end{aligned}
$$

and

$$
\begin{gathered}
\max \left\{\left\|x_{2 m(p)}-y_{2 n(p)}\right\|,\left\|x_{2 n(p)}-y_{2 m(p)}\right\|\right\} \\
\leq \max \left\{\left\|x_{2 m(p)}-x_{2 m(p)+2}\right\|+\left\|x_{2 m(p)+2}-y_{2 n(p)+2}\right\|+\left\|y_{2 n(p)+2}-y_{2 n(p)}\right\|,\right. \\
\left.\left\|x_{2 n(p)}-x_{2 n(p)+2}\right\|+\left\|x_{2 n(p)+2}-y_{2 m(p)+2}\right\|+\left\|y_{2 m(p)+2}-y_{2 m(p)}\right\|\right\} \\
\leq \max \left\{\left\|x_{2 m(p)+2}-y_{2 n(p)+2}\right\|,\left\|x_{2 n(p)+2}-y_{2 m(p)+2}\right\|\right\}+ \\
\max \left\{\left\|x_{2 m(p)}-x_{2 m(p)+2}\right\|+\left\|y_{2 n(p)+2}-y_{2 n(p)}\right\|,\right. \\
\left.\left\|x_{2 n(p)}-x_{2 n(p)+2}\right\|+\left\|y_{2 m(p)+2}-y_{2 m(p)}\right\|\right\} .
\end{gathered}
$$

Taking $p \longrightarrow \infty$ in the above two inequalities, and using (4.8) and (4.11), we conclude that

$$
\lim _{p \longrightarrow \infty} \max \left\{\left\|x_{2 m(p)+2}-y_{2 n(p)+2}\right\|,\left\|y_{2 m(p)+2}-x_{2 n(p)+2}\right\|\right\}=d(A, B)+\epsilon .
$$

Again

$$
\begin{aligned}
& \max \left\{\left\|x_{2 m(p)+2}-y_{2 n(p)+2}\right\|,\left\|y_{2 m(p)+2}-x_{2 n(p)+2}\right\|\right\} \\
& =\max \left\{\left\|F\left(y_{2 m(p)+1}, x_{2 m(p)+1}\right)-F\left(x_{2 n(p)+1}, y_{2 n(p)+1}\right)\right\|\right. \\
& \left.\quad\left\|F\left(x_{2 m(p)+1}, y_{2 m(p)+1}\right)-F\left(y_{2 n(p)+1}, x_{2 n(p)+1}\right)\right\|\right\} \\
& =\max \left\{\left\|F\left(x_{2 n(p)+1}, y_{2 n(p)+1}\right)-F\left(y_{2 m(p)+1}, x_{2 m(p)+1}\right)\right\|,\right. \\
& \leq \max \left\{k\left[\left\|x_{2 n(p)+1}-y_{2 n(p)+2}\right\|+\left\|y_{2 m(p)+1}-x_{2 m(p)+2}\right\|\right]+(1-2 k) d(A, B),\right. \\
& \left.\quad k\left[\left\|x_{2 m(p)+1}-y_{2 m(p)+2}\right\|+\left\|y_{2 n(p)+1}-x_{2 n(p)+2}\right\|\right]+(1-2 k) d(A, B)\right\}
\end{aligned}
$$

Taking $p \rightarrow \infty$, using (4.3) and (4.12), we get $d(A, B)+\epsilon \leq d(A, B)$, which is a contradiction. Therefore (4.9) holds. 
Since $\left\{x_{n}\right\}$ and $\left\{y_{n}\right\}$ are sequences in $A$ and $B$ respectively, we have

$$
d(A, B) \leq\left\|x_{2 m}-y_{2 n}\right\| \text { and } d(A, B) \leq\left\|y_{2 m}-x_{2 n}\right\| \text { for all } m, n,
$$

and hence

$$
d(A, B) \leq \max \left\{\left\|x_{2 m}-y_{2 n}\right\|,\left\|y_{2 m}-x_{2 n}\right\|\right\} \text { for all } m, n .
$$

Then from (4.9), we can write that given any $\epsilon>0$ there exists an integer $N$ such that for all $m, n>N$

$$
d(A, B)-\epsilon<d(A, B) \leq \max \left\{\left\|x_{2 m}-y_{2 n}\right\|,\left\|y_{2 m}-x_{2 n}\right\|\right\}<d(A, B)+\epsilon,
$$

which implies that for all $m, n>N$

$$
d(A, B)-\epsilon<d(A, B) \leq\left\|x_{2 m}-y_{2 n}\right\|<d(A, B)+\epsilon,
$$

and

$$
d(A, B)-\epsilon<d(A, B) \leq\left\|y_{2 m}-x_{2 n}\right\|<d(A, B)+\epsilon .
$$

Therefore,

$$
\lim _{m, n \longrightarrow \infty}\left\|x_{2 m}-y_{2 n}\right\|=d(A, B) \quad \text { and } \quad \lim _{m, n \longrightarrow \infty}\left\|y_{2 m}-x_{2 n}\right\|=d(A, B) .
$$

By (4.10), (4.13) and Lemma 2.3, we have

$$
\lim _{m, n \longrightarrow \infty}\left\|x_{2 m}-x_{2 n}\right\|=0 \quad \text { and } \quad \lim _{m, n \longrightarrow \infty}\left\|y_{2 m}-y_{2 n}\right\|=0,
$$

which implies that $\left\{x_{2 n}\right\}$ and $\left\{y_{2 n}\right\}$ are a Cauchy sequences in $A$ and $B$ respectively. The sets $A$ and $B$ being closed, there exist $u \in A$ and $v \in B$ such that

$$
x_{2 n} \longrightarrow u \text { and } y_{2 n} \longrightarrow v \text { as } n \longrightarrow \infty
$$

Since $F$ is coupling with respect to $A$ and $B$, and $u \in A, v \in B$, we have $F(u, v) \in B$. Then we have

$$
d(A, B) \leq\|u-F(u, v)\|
$$

Now

$$
\begin{aligned}
\|u-F(u, v)\| & \leq\left\|u-x_{2 n}\right\|+\left\|x_{2 n}-F(u, v)\right\| \\
& \leq\left\|u-x_{2 n}\right\|+\left\|F\left(y_{2 n-1}, x_{2 n-1}\right)-F(u, v)\right\| \\
& =\left\|u-x_{2 n}\right\|+\left\|F(u, v)-F\left(y_{2 n-1}, x_{2 n-1}\right)\right\| \\
& \leq\left\|u-x_{2 n}\right\|+k\left[\|u-F(u, v)\|+\left\|y_{2 n-1}-F\left(y_{2 n-1}, x_{2 n-1}\right)\right\|\right]+(1-2 k) d(A, B) \\
& \leq\left\|u-x_{2 n}\right\|+k\left[\|u-F(u, v)\|+\left\|y_{2 n-1}-x_{2 n}\right\|\right]+(1-2 k) d(A, B),
\end{aligned}
$$

which implies that

$$
\|u-F(u, v)\| \leq \frac{1}{1-k}\left\|u-x_{2 n}\right\|+\frac{k}{1-k}\left\|y_{2 n-1}-x_{2 n}\right\|+\left(1-\frac{k}{1-k}\right) d(A, B) .
$$


Taking $n \longrightarrow \infty$ in the above inequality, using (4.3) and (4.15), we have

$$
\|u-F(u, v)\| \leq \frac{k}{1-k} d(A, B)+\left(1-\frac{k}{1-k}\right) d(A, B)=d(A, B) .
$$

Combining (4.16) and (4.17), we have

$$
d(A, B) \leq\|u-F(u, v)\| \leq d(A, B)
$$

which implies $\|u-F(u, v)\|=d(A, B)$. Similarly, we can prove that $\|v-F(v, u)\|=d(A, B)$. Hence $(u, v)$ is a coupled best proximity point of $F$.

Conclusion. Coupled fixed point results are known for their applications. Here we have presented an instance of an application where the concept of coupled fixed points has been utilized in the solution of a minimization problem. The approaches adapted here is new to the category of problems considered. It appears that the present method can be further applied to similar problems through other types of couplings satisfying different inequalities. This can be treated as an open problem.

Acknowledgement: The work is supported by the Science and Engineering Research Board, Government of India, under Research Project No. PDF/2016/000353. The support is gratefully acknowledged.

\section{REFERENCES}

[1] A. Abkar, M. Gabeleh, Best proximity points for cyclic mappings in ordered metric spaces, J. Optim. Theory Appl. 151 (2011), 418-424.

[2] A. Abkar, M. Gabeleh, The existence of best proximity points for multivalued non-self-mappings, Rev. R. Acad. Cienc. Exactas Fs. Nat., Ser. A Mat., RACSAM, 107 (2013), 319-325.

[3] R. P. Agarwal, D. O‘Regan, D. R. Sahu, Fixed Point Theory for Lipschitzian-Type Mappings with Applications, Springer, New York, 2009.

[4] I. Altun, D. Turkoglu, Some fixed point theorems for weakly compatible multivalued mappings satisfying an implicit relation, Filomat 22 (2008), 13-21.

[5] M. A. Ahmed, Common fixed point theorems for weakly compatible mappings, Rocky Mountain J. Math. 33 (2003), 1189-1203.

[6] C. D. Bari, T. Suzuki, C. Vetro, Best proximity points for cyclic Meir - Keeler contractions, Nonlinear Anal. 69 (2008), 3790-3794.

[7] A. Bejenaru, A. Pitea, Fixed point and best proximity point theorems in partial metric spaces, J. Math. Anal. 7(4) (2016), $25-44$.

[8] B. S. Choudhury, P. Maity, P. Konar, A global optimality result using nonself mappings, Opsearch 51(2) (2014), 312-320.

[9] B. S. Choudhury, N. Metiya, P. Maity, Coincidence point results of multivalued weak C-contractions on metric spaces with a partial order, J. Nonlinear Sci. Appl. 6 (2013), 7-17.

[10] B. S. Choudhury, P. Maity, Cyclic coupled fixed point result using Kannan type contractions, J. Operators 2014 (2014), Art. ID 876749,5 pages.

[11] B. S. Choudhury, P. Maity, Best proximity point results in generalized metric spaces, Vietnam J. Math. 44 (2016), 339-349. 
[12] B. S. Choudhury, P. Maity, N. Metiya, Best proximity point results in setvalued analysis, Nonlinear Anal. Modelling Control. 21 (2016), 293-305.

[13] B. S. Choudhury, P. Maity, K. Sadarangani, A best proximity point theorem using discontinuous functions, J. convex anal. 24 (2017), 41-53.

[14] B. Damjanovic, D. Dragan, Multivalued generalizations of the Kannan fixed point theorem, Filomat 25(1) (2011), $125-131$.

[15] A. A. Eldred, P. Veeramani, Existence and convergence of best proximity points, J. Math. Anal. Appl. 323 (2006), 10011006.

[16] E. Karapinar, I. M. Erhan, Best proximity point on different type contractions, Appl. Math. Info. Sci. 5(3) (2011), 558-569.

[17] B. Fisher, Common fixed points of mappings and setvalued mappings, Rostock Math. Colloq. 18 (1981), 69-77.

[18] M. Gabeleh, Best proximity points: global minimization of multivalued non-self mappings, Optim. Lett. 8 (2014), 11011112.

[19] J. Gornicki, Fixed point theorems for Kannan type mappings, J. Fixed Point Theory Appl. 19 (3) (2017), $2145-2152$.

[20] A. Ilchev, B. Zlatanov, Error estimates for approximation of coupled best proximity points for cyclic contractive maps, Appl. Math. Comput. 290 (2016), 412-425.

[21] R. Kannan, Some result on fixed points, Bull. Calcutta Math. Soc. 60 (1968), 71-76.

[22] R. Kannan, Some result on fixed points-II, Amer. Math. Monthly 76 (1969), 405-408.

[23] M. Petric, Best proximity point theorems for weak cyclic Kannan contraction, Filomat 25(1) (2011), 145-154.

[24] V. Pragadeeswarar, M. Marudai, P. Kumam, Best proximity point theorems for multivalued mappings on partially ordered metric spaces, Nonlinear Sci. Appl. 9 (2016), 1911-1921.

[25] W. Shatanawi, A. Pitea, Best proximity point and best proximity coupled point in a complete metric space with (P)property, Filomat 29(1) (2015), 63-74.

[26] T. Suzuki, M. Kikkawa, C. Vetro, The existence of best proximity points in metric spaces with the property UC, Nonlinear Anal. 71 (2009), 2918-2926. 